\title{
ANALYSIS OF AN ARTHROPODAL SYSTEM FOR DESIGN OF A CLIMBING ROBOT
}

\author{
Austin Lozano*, Gregory Peters, and Dikai Liu \\ Centre for Autonomous Systems (CAS), University of Technology, Sydney (UTS), NSW 2007, Australia \\ *Corresponding author (austinlozano@gmail.com, Gregory.Peters@uts.edu.au,Dikai.Liu@uts.edu.au)
}

\begin{abstract}
This paper will discuss research on ant locomotion, especially in climbing adaptability and transversing steel bridge configurations, for the design of a climbing robot for inspection and condition assessment of complex steel bridge structures. Experiments in ant locomotion were performed and captured using high-speed video equipment capable of recording at 1000fps. Analysis of the data found that certain body postures in ants are adopted before proceeding with their intended direction, such as when climbing a vertical obstacle. Information on ant body posture, compliance and movement in climbing will also be presented. Furthermore, the working volumes of ant leg pairs are determined through computer aided analysis, which provides essential data for determining possible ant foot positions for locomotion. Discussions on how the findings can be used in designing a climbing robot is also presented.
\end{abstract}

Keywords: Climbing Robot, Steel Bridge Inspection, Arthropod, Arthropodal, Locomotion, Biomimicry

\section{INTRODUCTION}

Robots have traditionally been used to meet the ever increasing demands of production efficiency and cost savings. However occupational health and safety has been driving the development of a new range of robots to work in environments that are dangerous or impossible for humans to work safely. One such application is the periodical inspection for rust, paint degradation and maintenance needs on steel bridges. This inspection is a vital part of prolonging the life of the structure and ensuring public safety as well as minimising maintenance costs. The precarious height as well as the constant presence of vehicles provides a significant danger both to bridge workers and users during maintenance and inspection work.

There is an estimated 42,000 steel bridges in the European Union, more than 270000 in Japan and 210,000 in the United States [12]. The highly complex nature of steel bridge structures makes inspection difficult. Furthermore, the surface is typically unsmooth, with large rivets, bolts, flanges and varied layers of paint. Robotic bridge inspection requires robots be able to move inverted, over and past obstacles that might be encountered. A significant challenge facing deployment of climbing inspection robots is achieving reliable locomotion in this environment.

Recently a UTS research group developed a proof-ofconcept prototype quadrupedal robot; called the Reconfigurable Vertical Climber (RVC), with the intention of performing steel bridge maintenance exercises [10]. This prototype robot demonstrated feasibility, however a lot of challenges have been identified, and further research and design is required for future development of this concept.

\subsection{Motivation \& Requirements}

The success of steel bridge inspection by robots is determined by the robot's ability to effectively and safely navigate a complex bridge structure which consists of a lot of truss and frame structures, intersections, girders etc.. There is currently no robot capable of reliable navigation required in these complex environments [10], although robots for steel bridge maintenance (e.g., grit-blasting robot) have been developed $[11,13]$. Aiming at developing a biologically inspired climbing robot for inspection of complex steel bridges, this research is to understand how a biological system, such as arthropods, climbs and transfers between different surfaces. The ant was selected for analysis as they are known for capability and consistent 
manoeuvrability throughout entire structures. This research provides information of an arthropod's locomotion ability and techniques which could then be effectively implemented to climbing robots.

Efficient control logic and mechanical locomotion ability have significant effect on the success of a climbing robot. Analysis of arthropodal locomotion will enable novel design of robotic climbing systems.

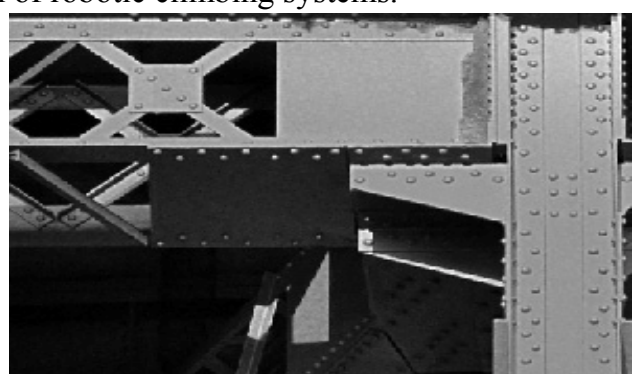

Fig. 1 An example steel bridge environment where inspection and condition assessment is required.

The environment shown in Fig. 1 is a typical example of complex steel bridge structure which consists of various angles, surfaces and structural members that a climbing robot should be able to safely, precisely and effectively navigate through. In some steel bridge structures, close proximity of rivet heads presents another issue the design must address.

Arthropods have developed a wide range of movement capabilities. They are able to move proficiently in very complex terrain. Fig. 2 shows an ant moving through a two-stage obstacle. If these can be adopted by climbing robots, it would be beneficial to the robot's climbing capabilities.

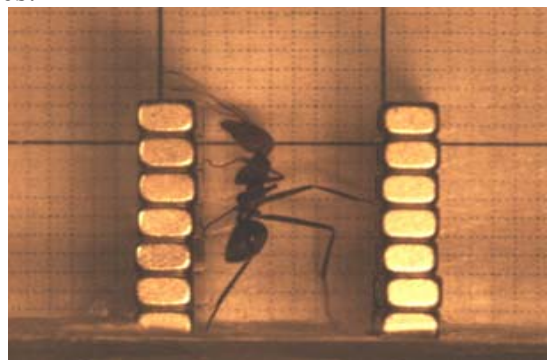

Fig. 2 Ant climbing an obstacle

\subsection{Current State of the Art}

The challenge of robotic climbing and locomotion on relatively smooth surfaces has received a lot of attention from researchers $[2,3,4,6,10,14,15]$. Adhesion principles have been studied $[1,10]$. Tracked systems such as Cleanbot II [17] provide improved performance in some aspects compared to other robots such as magnetic wall climbing robot [16]. However, a highly complex environment such as a steel bridge which has 90 degree plane transitions provides significant limitations on the success of a robotic climbing system. Alternatively frame walking robots such as the WALLY robot [18] usually involve a four-bar linkage design or rectangular hinged frames which translate the entire body with each step.

A robot with four or more legs is suited to climb complex environments. However the control complexity, power consumption and weight are increased [5].

The existing robotic systems are found to be difficult to meet the requirements of climbing complex steel bridge structures, and thus a biologically inspired approach is considered. Many walking robots are based on arthropod designs which typically feature inherently efficient movement and rapid adjustment to perturbations [6]. In robotic design, there exists general requirement for agile locomotion in a range of environments [6]: 'An agile robot must one, have inherently efficient movement; two, be able to generate rapid adjustments to transient perturbations and changes in load; and three, be able to take in sensory information and use it to alter direction of movement in order to orient toward goals and away from threats and to get over, under or around barriers'.

In this research, the Australian Meat Ant (Myrmex Purpureus) is chosen for study of locomotion, leg working volume, and leg and body compliancy and coordination. The goals of this study are to identify the following key criteria:

- Why and how a flexible body is used

- Overlapping working volumes of the legs

- The hierarchy of compliant structures

- Transfer from one surface to another

The findings will be discussed in the remainder of the paper, followed by discussion of the results.

\section{APPARATUS \& THE EXPERIMENT SETUP}

The ants were contained within a clear Perspex enclosure that is $100 \mathrm{~mm}$ in length, $20 \mathrm{~mm}$ high and $20 \mathrm{~mm}$ deep. This small enclosure provides a confined space to allow capture 
of walking gaits and surface transitions.

The ants were filmed using a high speed camera (150fps 1000fps) fitted with a macro lens with limited depth of field. The enclosure was illuminated with high intensity lighting. The rear wall of the enclosure was fitted with a $1 \mathrm{~mm}$ square grid pattern for measurements. Small neodymium magnets $(6.9 \times 2.6 \times 1.8 \mathrm{~mm})$ were positioned in various forms to generate a range of obstacles.

The ants were individually placed in the enclosure and allowed to explore the environment. Fig. 3 shows the experiment setup.

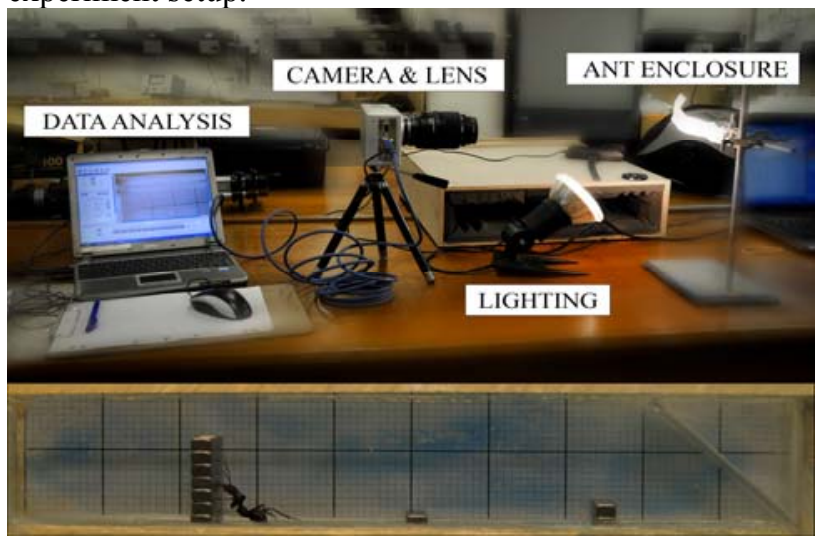

Fig. 3 Experimental setup of the equipment and Ant locomotion study enclosure.

\section{BODY FLEXIBILITY}

Many existing quadruped and hexapod robots have all legs rigidly mounted to a common solid platform. Often this common platform forms the body. An ant's body is made up of 3 distinct body segments; the head, the thorax and the gaster. The joint linking the gaster and thorax segment is a singular node or 2 segmented nodes depending on the ant species. The node and neck, which connects the head to the thorax, can be simplified to a 3 Degree of Freedom (DOF) joint which helps the ant maintain stability and movement through complex environments.

Ants were filmed while they were climbing over a $12.6 \mathrm{~mm}$ high wall and $3.6 \mathrm{~mm}$ bump. Each body segment was studied during locomotion. The study showed that ants flex their body segments to achieve stability, complex leg positions and further environment awareness. Frame ' $a$ ', 'b', 'c' and 'd' in Fig. 4 show the ant at different positions during its movement through a complex environment. The frames highlight the ant's flexibility in its three body sections. Blue line highlights the head, purple line highlights the middle thorax section, while green line highlights the rear abdomen section. The head of the ant is used for sensing the surrounding environment with the 3 DOF present in the neck giving the ant greater control in doing so. It is worth noting that the gaster, or the rear segment, tends to be positioned very close to the substrate. At times the ant uses the gaster similar to an extra leg (or tail) to keep the body above the substrate or to help position the body before climbing up a wall. The frames ' $a$ ' and ' $b$ ' in Fig. 5 show the ant in preparation to scaling the vertical wall. Corresponding frames 'c' and 'd', highlight flexibility the ant's body sections in the same manner as Fig. 4. Pay particular attention to the rear green abdomen section as the ant utilises it to assist maintain balance in order to climb the wall.

This segmented body flexibility has also been shown to change the centre of mass in the body, vital for active stability [7].

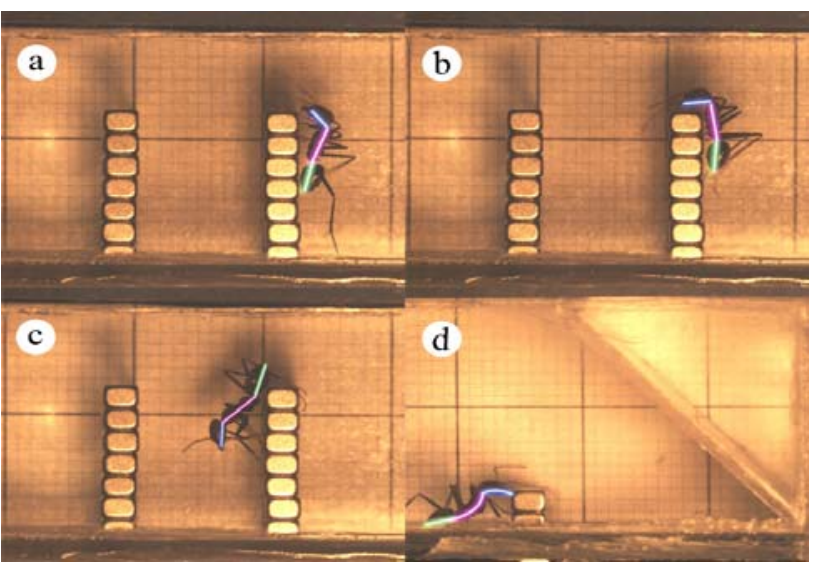

Fig. 4 Body flexibility in ants during locomotion.

\section{LEG WORKING VOLUMES}

Arthropodal systems have multiple legs with different size, which is different from most climbing robots that have multiple legs with the same size. For example, an ant has six different sized legs. These legs are in pairs and have different working volumes. There are three leg pairs within the ant. These leg pairs are the front pair, the middle pair, and the rear pair. Through experimental observations of the ant's legs, the left and right side of ant leg pairs are identical in length and working angle. The measured tolerance of the ant legs was within $2 \%$ of each other. The 
front, middle and rear legs are different in length. Tab. 1 shows that the front leg is about $79 \%$ the length of the middle leg and about $59 \%$ of the rear leg while the middle leg is about $75 \%$ the length of the rear leg. In the same ant species, the leg length ratios are similar. A sample of 12 ants was used for this analysis. Shown in Tab. 1 are multiplier values for determining total length of all leg pairs given a single leg segment length.

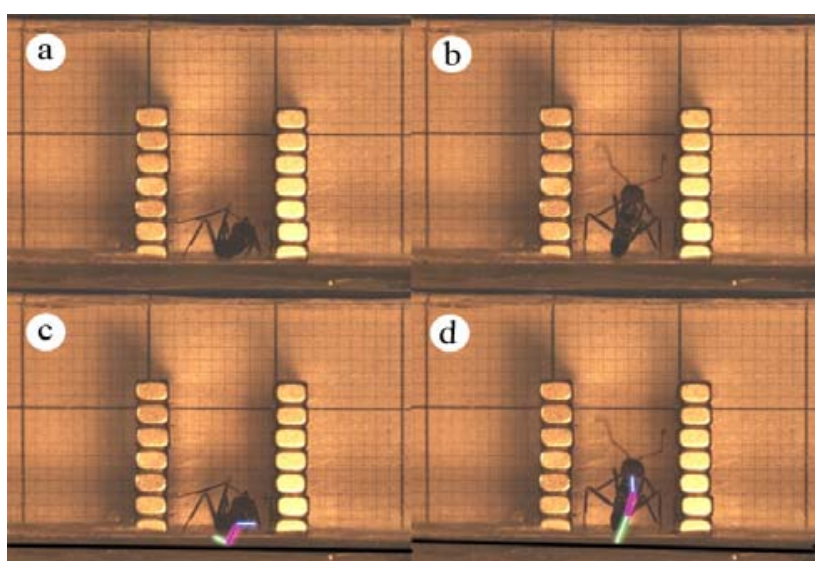

Fig. 5 Ant utilises gaster to position body in a rearing position before climbing.

\begin{tabular}{|c|c|c|c|}
\hline Given Leg Segment Length & Front & Middle & Rear \\
\hline Front Coxa Length = 1unit & 9.64 & 12.21 & 16.33 \\
\hline Front Femur Length = 1 unit & 2.98 & 3.77 & 5.04 \\
\hline Front Tibia Length = 1 unit & 3.49 & 4.41 & 5.90 \\
\hline Front Tarsi Length = 1 unit & 3.79 & 4.78 & 6.39 \\
\hline \hline Middle CoxaLength = 1 unit & 11.34 & 14.13 & 18.96 \\
\hline Middle Femur Length = 1 unit & 2.44 & 3.04 & 4.08 \\
\hline Middle Tibia Length = 1 unit & 2.91 & 3.62 & 4.86 \\
\hline \hline Middle Tarsi Length = 1 unit & 1.92 & 3.10 & 4.16 \\
\hline Rear Coxa Length = 1 unit & 9.23 & 11.55 & 15.38 \\
\hline Rear Femur Length = 1 unit & 1.81 & 2.27 & 3.02 \\
\hline Rear Tibia Length = 1 unit & 2.24 & 2.80 & 3.73 \\
\hline Rear Tarsi Length = 1 unit & 1.82 & 2.28 & 3.04 \\
\hline
\end{tabular}

Tab. 1. Multiplier for determining leg lengths.

These results allow proportional biomimicry of ant leg structures in climbing robot design. For example, a robot which needs to be designed with front leg lengths of $100 \mathrm{~mm}$ means that the middle leg lengths is about $127 \mathrm{~mm}$ $[(12.21 / 9.64) * 100]$ while the rear leg lengths is about $169 \mathrm{~mm}[(16.33 / 9.64) * 100]$. In order to determine the lengths of each individual leg segment, we can also use Tab. 1. For example, because we used the first row to determine the length of the other leg pairs, we can calculate the length of the front coxa which is about $10 \mathrm{~mm}$ [(1/9.64) * 100]. From here we can calculate the remaining segment lengths using the rear leg ratio (chosen for measurement accuracy as it's the longest leg). For example the front femur length is about $33 \mathrm{~mm}$ [(16.33/5.9) * 10].

Therefore different configurations of climbing robot leg lengths can be designed to replicate an ant's locomotion, with consideration of the specific application environments of a climbing robot. A simulation environment needs to be built in order to conduct comprehensive study on selection of robot leg length for different application environments.

Determining the working volume of an ant leg requires information on the movement ability of the leg. From the analysis of ant leg movement based on the high-speed video captures, it is found that the ranges of motion for each leg and leg segment are determined as shown in Fig. 6 and Tab. 2.
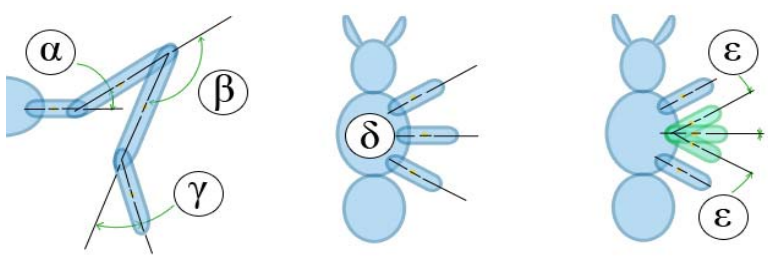

Fig. 6 Ant leg range of motion.

\begin{tabular}{|c|c|c|l|}
\hline \multicolumn{2}{|c|}{ Joint } & Motion & \multicolumn{1}{|c|}{ Note } \\
\hline$\alpha$ & Coxa-Femur & $+60^{\circ}$ & Positive pitch of $60^{\circ}$ \\
\hline$\beta$ & Tibia-Tarsal & $\pm 5^{\circ}$ & Passive flex segment \\
\hline$\gamma$ & Femur-Tibia & $-145^{\circ}$ & Negative pitch of $145^{\circ}$ \\
\hline$\delta$ & Coxa Positions & $\pm 45^{\circ}, 0^{\circ}$ & $\begin{array}{l}\text { Coxa position at rest } \\
\text { (standing) }\end{array}$ \\
\hline$\varepsilon$ & Body-Coxa & $\pm 45^{\circ}$ & $\begin{array}{l}\text { Coxa can rotate } 45^{\circ} \text { from its } \\
\text { original position in x-y plane }\end{array}$ \\
\hline
\end{tabular}

Tab. 2 Ranges of motion of ant leg segments

By varying leg segment positions within the upper and lower angle limits (based on Tab. 2), leg extremity position can be plotted. Consequently, an approximate working volume for each leg is built as shown in Fig. 7.

\section{HIERARCHY OF COMPLIANT STRUCTURES}

Leg design of a climbing robot is very challenging. Information and understanding on ant body and leg compliance would be helpful in robot leg design.

It is found that the pre-tarsus segment on all leg pairs of an ant, provide a significant amount of compliance in the ant leg. When climbing over bumps, vertical sections and ceilings, the ant body and legs will, to an extent, naturally 
bend to the contours of the environment. This attribute helps insects retain correct posture required for agile locomotion. The ant's tarsus is observed to maintain different stiffness attributes depending on the length of the tarsus and the distance from the tibia-tarsus joint. Shown in Fig. 8 is the observed relationship between the inherent compliancy of the legs to the distance from the tibia segment.

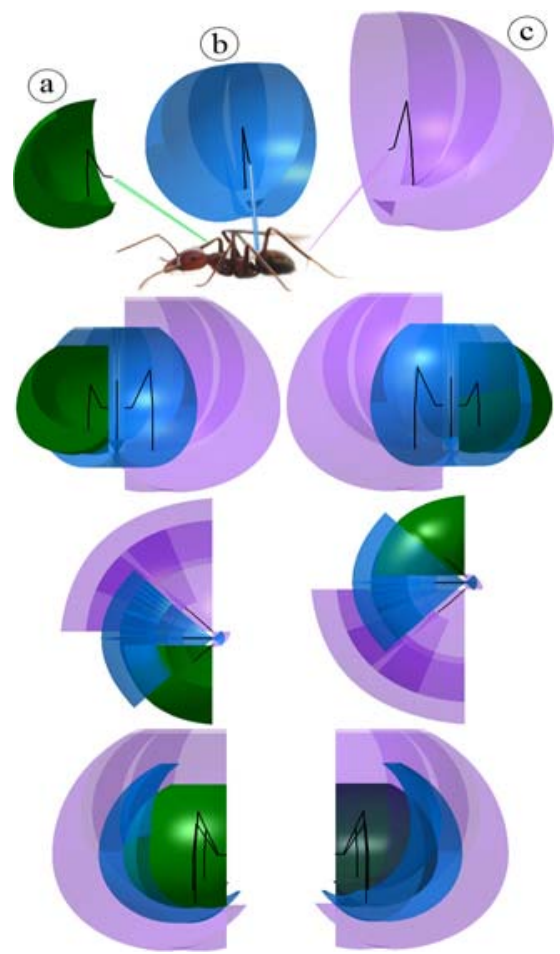

Fig. 7 Overlapping working volumes of the ant's legs top picture, (a) green, front leg; (b) blue, middle leg; (c) purple, rear leg); 2nd row: inner and outer side views; 3rd row: top and bottom view; 4th row: front and back view.

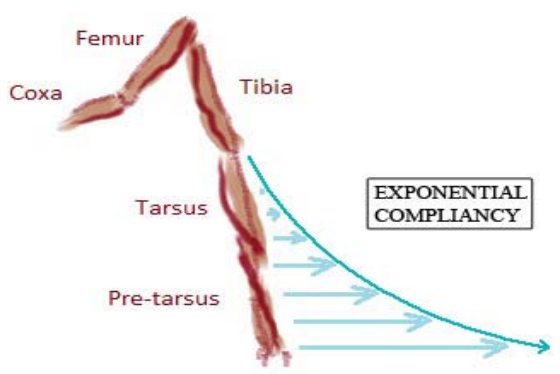

Fig. 8 Ant leg compliancy observed as exponential

Ant body segments provide compliancy and change in centre of gravity. Although the tarsus has natural flex, a muscle controls the tendon reaching from the tibia through to the foot of the leg. 'This tripartite muscle is located in the femur and tibia and pulls on a long tendon-like apodeme that reaches out into the pre-tarsus' [8]. This tendon allows for pull and thrust actions leading to contraction and expansion of the tarsus and pre-tarsus. This tendon grants the ant the ability to readily control the compliancy of the legs by restricting the amount of flex. Furthermore, it was observed through experimentation [8] that the tendon will let the tarsus sections 'curl' and act in a similar way to the fingers when grabbing an item. The ant utilises this attribute when climbing over edges. Through experimentation, the ant was observed to 'grab' the edge of a wall with its rear leg tarsus sections; acting similar to an anchor point. This allows the ant to 'roll' its body position; and hence its centre of gravity, over to the opposite side of the wall. This motion was observed in the ant study and can be viewed in Fig. 9.

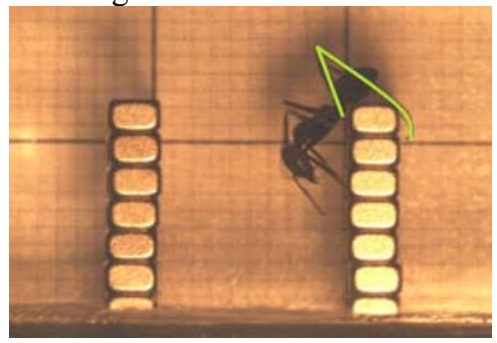

Fig. 9 Ant uses compliance to aid in passing an obstacle. The three main sections of the ant body (head, thorax and abdomen) are utilised differently. The head is used for communicating, environment sensing and eating. The thorax has all 6 legs attached, while the gaster holds the food. In order to mimic ant locomotion, the legs should be placed on the same section of body. Since ants have tendons to vary the degree to which the legs will comply with the environment. The body of the ant is able to remain stable above the substrate.

\section{SURFACE TRANSITION}

Most hexapod robots previously designed do not have varied and overlapping work volumes, the walking gaits and any surface transition behaviours do not truly mimic the ant. In order to develop leg motion planning algorithms, body and leg coordination approaches and control protocols for a hexapod robot to climb various surfaces/structural members in a steel bridge setting, ants 
were studied performing various surface transitions. Fig. 10 show locomotion capture environments
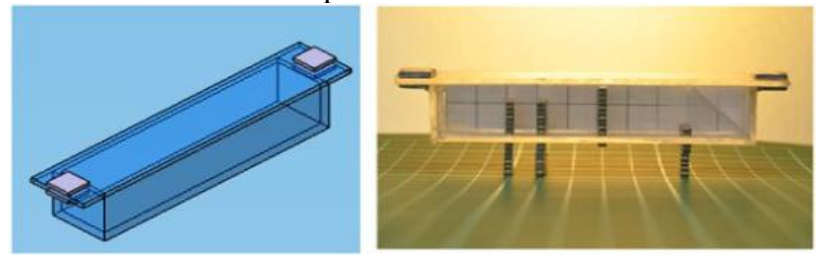

Fig. 10 Transition experimental enclosure

It is found that normal and slow movements by ants utilise individual leg sequences. Ants also perform tetrapod gait, which has previously been proven to be undesirable in robotic applications in respect to stability, speed and power consumption [9]. At high speeds, ants adopt the tripod gait. Locomotion through a complex environment which is expected from a capable climbing robot is replicated on the climbing ant experiments. 72 transition sequences through 21 transitions were captured using the high speed camera.

Observations of ants transitioning from a horizontal to a vertical surface demonstrate that ants assume a rearing stance. The front legs and middle legs are placed onto the vertical surface whilst the rear legs compress to prepare to push the body upwards. This sequence is shown in Fig. 11 through the 11 frames detailing step by step, the ant's method in scaling the wall from a horizontal rest position.

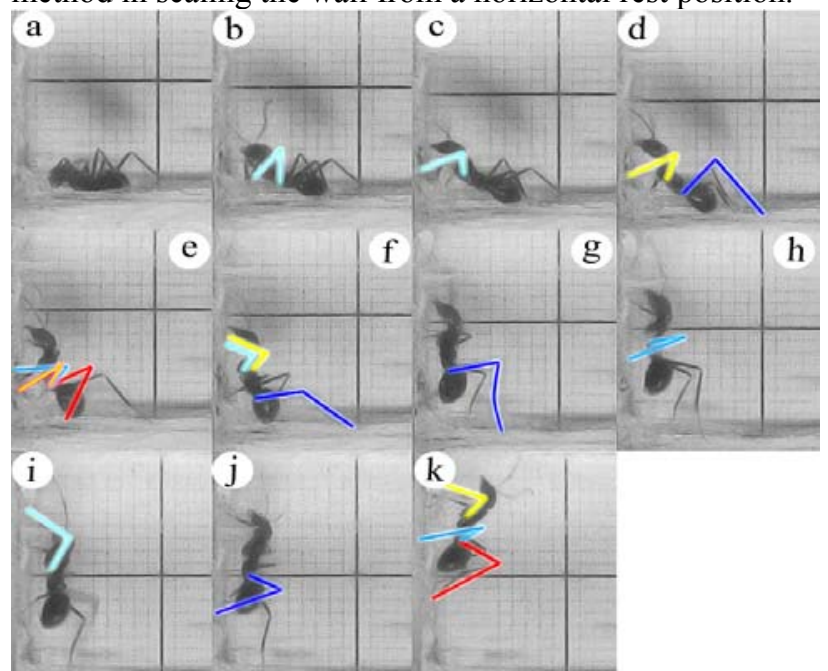

Fig. $11^{1}$ Surface transition from horizontal to vertical. (a)

\footnotetext{
${ }^{1}$ Leg abbreviations for Fig. 11 include L1 [aqua blue], left front leg; L2 [cerulean blue], left middle leg; L3 [dark blue], left rear leg; R1 [yellow], right front leg; R2 [peach], right middle leg; and R3 [red], right rear leg.
}

Ant prepares the transition; (b) L1 prepares; (c) L1 reaches vertical surface; (d) R1 \& L3 move simultaneously. R1 attaches to vertical wall (e) R2, L2, R3 move as one. Middle pair attaches to vertical surface \& R3 compresses for rearing; (f) Front pair reach up surface \& L3 starts compressing; (g) L3 moves close to vertical surface while ant pulls body up wall using front legs; (h) L2 moves further up vertical surface; (i) Ant reaches further up by moving L1 while pushing with R3; (j) Ant places L3 onto vertical surface while pushing with R3; (k) Ant brings R3 onto vertical surface. L2 \& R1 move simultaneously up.

\section{DISCUSSION \& FUTURE WORK}

This research on ant locomotion provides robot builders with design and control attributes inherent in climbing ants. Ant climbing gait sequences, leg compliance, leg length ratios, leg working volumes, and leg and body coordination provide useful information for design of robots to climb complex structural environments. The relative leg sections' lengths together with their working angles provide the legs working envelop.

When attempting to replicate an ant's locomotion ability into a climbing robot, problems in robot design and control will be encountered. One problem is the robot leg design including the length of segments (joints and links), the number and capacity of actuators. This problem in turn leads to problems such as increased weight, on board power requirements and processing time. Design concepts based on the information presented in this paper should be evaluated against other designs to ensure the requirements of the robot are met.

From the knowledge gained on locomotion in complex environments, it would be highly recommendable that path planning logic be further studied to gain a deeper understanding required for climbing robot design. Coordination of the body and legs will dictate success. It is noted that even ants are susceptible to falling off their intended direction of motion. Arthropods adjust rapidly to complex environments and unexpected circumstances. This was shown through the conducted experiments when the ant was observed to rapidly recover from a fall. The ant landed upside down on the horizontal surface and recovered back to its original rest position within 100 
milliseconds. Throughout the whole fall and recovery sequence, the ant's body does not hit the substrate. This is because the ant retracts its legs which took the majority of the force of the fall. It is recommended that robot control for recovering from a fall should be further researched. This is where information in ant recovery sequences will be useful as a countermeasure for falling climbing robots.

\section{ACKNOWLEDGMENTS}

This work is supported by the ARC Linkage Grant (ARCLP100200750), by the Roads and Traffic Authority (RTA) NSW, and the University of Technology (UTS), Sydney.

\section{REFERENCES}

[1] Longo, D. \& Muscato, G, 'Adhesion techniques for climbing robots: State of the art and experimental considerations', paper presented to the 11th International Conference on Climbing and Walking Robots and the Support Technologies for Mobile Machines, Coimbra, Portugal, 11/11/2008.

[2] Ross, B., Bares, J. \& Fromme, C, 'A Semi-Autonomous Robot for Stripping Paint from Large Vessels', The International Journal of Robotics Research, vol. 22, no. 78, pp. 617-626, 2003

[3] Hillenbrand, C., Schmidt, D. \& Berns, K., 'CROMSCI A Climbing Robot With Multiple Sucking Chambers For Inspections Tasks', WSPC, Robotics Research Lab, Department of Computer Science, University of Kaiserslautern, Germany , 2008

[4] Bonaccorso, F., Bruno, C., Longo, D. \& Muscato, G, 'Structure and Model Identification of a vortex based suction cup', 11th International Conference on Climbing and Walking Robots and the Support Technologies for Mobile Machines, Coimbra, Portugal, 2008

[5] Weihua, S., Hongjun, C. \& Ning, X, 'Navigating a Miniature Crawler Robot for Engineered Structure Inspection', Automation Science and Engineering, IEEE Transactions on, vol. 5, no. 2, pp. 368-373, 2008

[6] Ritzmann, R., Gorb, S \& Quinn, R, 'Arthropod locomotion systems: from biological materials and systems to robotics', Arthropod Structure \& Development, vol. 33, no. 3, pp. 183-185, 2004

[7] Watson, J., Ritzmann, R et al, 'Control of obstacle climbing in the cockroach, Blaberus discoidalis', 2002

[8] Federle, W.E., 'Locomotion and adhesion: dynamic control of adhesive surface contact in ants', Arthropod
Structure \& Development, vol. 33, no. 1, pp. 67-75, 2003 [9] Santos, G., Jimenez, M \& Armada, A, 'Dynamic Effects in Statically Stable Walking Machines', Journal of Intelligent \& Robotic Systems, vol.23, no.1, pp.71-85, 1998 [10] G. Peters, D. Pagano, D.K. Liu and K. Waldron (2010), "A Prototype Climbing Robot for Inspection of Complex Ferrous Structures", Proceedings of the 13th International Conference on Climbing and Walking Robots and the Support Technologies for Mobile Machines (CLAWAR'2010), pp150-156, 31 August - 03 September 2010, Nagoya, Japan

[11] D.K. Liu, G. Dissanayake, P.B. Manamperi, P.A. Brooks, G. Fang, G. Paul, S. Webb, N. Kirchner, P. Chotiprayanakul, N. M. Kwok, T.R. Ren (2008), “A robotic system for steel bridge maintenance: research challenges and system design", Proceedings of the Australasian Conference on Robotics and Automation (ACRA 08), 3-5 Dec 2008, Canberra, Australia, 7 pages.

[12] Balaguer, C., Gimenez, A. \& Jardon, A. 2005, 'Climbing Robots' Mobility for Inspection and Maintenance of 3D Complex Environments', Autonomous Robots, vol. 18, no. 2 - March 2005, 99. 157-169.

[13] G. Paul, D.K. Liu, N. Kirchner and G. Dissanayake (2009), "An effective approach to simultaneous mapping and surface-type identification of complex $3 \mathrm{~d}$ environments", Journal of Field Robotics, 26 (11-12): pp915-933, November-December 2009;

[14] Kingsley, D., Quinn, R \& Ritzmann, R 2003, A Cockroach Inspired Robot With Artificial Muscles, Case Western Reserve University, Cleveland Ohio.

[15] Dzhantimirov, P., et al. 2007, HIL/SIL by development of six-legged robot Slair2, Institute for Electrical Energy Systems, University of Magdeburg, Magdeburg, Germany.

[16] Fischer, W., Tache, F. \& Siegwart, R. 2008, 'Magnetic Wall Climbing Robot for Thin Surfaces with Specific Obstacles', Field and Serivce Robotics, vol.42, pp. 551-561

[17] Zhu J.; Sun D.; Tso S-K, "Development of a tracked Climbing Robot", Journal of Intelligent and Robotic Systems, Vol. 35, N.4, Dec. 2002, pp.427-443.

[18] G. Muscato, G. Trovato, "Motion control of a pneumatic climbing robot by means of a fuzzy processor", First international symposium CLAWAR '98 Climbing and Walking Robots, Brussels, 26-28 Novembre 1998. 